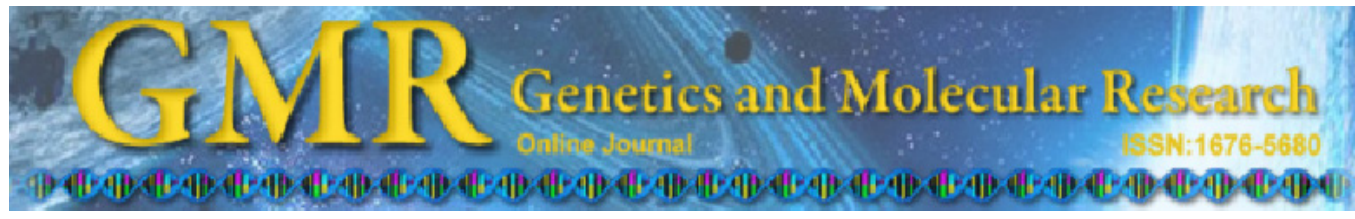

\title{
Differential gene expression and functional analysis of pit cells from regenerating rat liver
}

\author{
C.S. Xu ${ }^{1,2}$, X.G. Chen ${ }^{2}$, C.F. Chang ${ }^{2}$, G.P. Wang ${ }^{1}$, W.B. Wang ${ }^{2}$, L.X. Zhang ${ }^{1}$, \\ Q.S. Zhu' ${ }^{1}$ and L. Wang ${ }^{1}$ \\ ${ }^{1}$ College of Life Science, Henan Normal University, Xinxiang, China \\ ${ }^{2}$ Key Laboratory for Cell Differentiation Regulation, \\ Henan Normal University, Xinxiang, China \\ Correspondence author: C.S. Xu \\ E-mail: cellkeylab@126.com
}

Genet. Mol. Res. 10 (2): 678-692 (2011)

Received September 3, 2010

Accepted November 16, 2010

Published April 19, 2011

DOI 10.4238/vol10-2gmr1052

\begin{abstract}
Hepatic pit cells are a population of large granular lymphocytes that substantially contribute to hepatic immunity. Studies have proven that pit cells have a role in liver regeneration, but the details of the relationship between pit cells and liver regeneration is not clear at present. We subjected rats to a two-third hepatectomy; pit cells with high purity were obtained with Percoll density centrifugation and immunomagnetic bead methods, and the changes in mRNA levels in pit cells from the regenerating liver were monitored up to $168 \mathrm{~h}$ using a Rat Genome 2302.0 Array composed of 25,020 distinct rat liver cDNA clones. Of the 25,020 genes analyzed, 612 known and 358 unknown genes were identified to be associated with liver regeneration. The 612 known genes are classified into up-regulation and down-regulation patterns based on the expression levels; they primarily participate in at least 23 biological activities based on gene ontology analysis. Together with gene function enrichment analysis, cytokines and a growth factor-mediated pathway in pit cells were activated at an early phase of liver regeneration; pit cell proliferation occurred from 24-72 $\mathrm{h}$ after liver hepatectomy; the machinery of pit cell differentiation commenced early and came into play late; an immune/inflammatory response was enhanced late. Expression pattern analysis of functionally
\end{abstract}


classified genes in pit cells can give insights into the relationship between pit cells and liver regeneration.

Key words: Partial hepatectomy; Rat liver regeneration; Pit cells; Gene expression

\section{INTRODUCTION}

Pit cells, morphologically defined as large granular lymphocytes and functionally as liver-associated natural killer cells, are located inside sinusoidal lumina, where they adhere to endothelial cells and Kupffer cells (Nakatani et al., 2004). Pit cells are the major component of the innate immune system in the liver and substantially contribute to hepatic immunity (Paschos et al., 2008). Experimental studies have shown that pit cells represent the first line of liver defense response, in collaboration with Kupffer cells, through various mechanisms, including secreting interferon- $\gamma$ (IFN- $\gamma$ ), tumor necrosis factors (TNF), colony-stimulating factors, perforin and granzyme, and so on (Griffini et al., 1996). There are also some studies demonstrating that this population of natural killer cells are the important regulators of liver regeneration caused by physical or chemical stimuli such as $2 / 3$ hepatectomy, and can affect the turnover of hepatocytes by producing positive or negative signals (Kmieć, 2001). Studies carried out by Francavilia et al. (1997) found that the number of pit cells was restored on day 3 after partial hepatectomy, but its spontaneous killing functions were continuously repressed throughout the period of liver regeneration, while the in vivo depletion of pit cells resulted in the significant augmentation of liver regeneration, which suggested the involvement of pit cells in regulating the extent of regeneration. Findings from experiments by Wei et al. (2010) showed that activation of pit cells can inhibit liver regeneration via a TNF-dependent mechanism in the toxin-induced liver injury model in mice, while depletion of pit cells could allow for the restoration of regeneration. Observations from other studies showed that pit cells also could stimulate oval cell-dependent hepatic restoration by releasing inflammatory factors, such as TNF- $\alpha$, IFN- $\gamma$, which is identified as a negative regulator of liver regeneration in rats (Swain, 2008). Seemingly, the relationship between pit cells and liver regeneration is extremely complicated. Although the role of pit cells in hepatic reconstitution has been investigated, so far, there are very few studies comprehensively addressing its relevance to liver regeneration at a transcriptional level. To determine the role of pit cells in liver regeneration, we obtained pit cells with high purity, then measured the temporal expression profiles of pit cells after partial hepatectomy in rats using Rat Genome 230 2.0 Array (Wang et al., 2009), and identified the major clusters of up- and down-regulated genes that were further analyzed by methods in Systems Biology.

\section{MATERIAL AND METHODS}

\section{Preparation of the rat partial hepatectomy model}

Healthy Sprague-Dawley rats, weighing $190 \pm 20$ g, were provided by the Experimental Animal Center of Henan Normal University under normal conditions. A total of 114 adult rats were randomly divided into 19 groups of 6 rats each: 9 partial hepatectomy groups, 9 
sham-operated groups and one control group. After ether anesthesia, the rats in partial hepatectomy groups underwent $70 \%$ partial liver resection according to a previously described protocol (Higgins and Anderson, 1931). The partially hepatectomized rats were allowed free access to food and water for $2,6,12,24,30,36,72,120$, and $168 \mathrm{~h}$, respectively, until liver perfusion experiments were conducted. For the rats in sham-operated groups, the surgical operation was done as for the partial hepatectomy, but the liver was not removed. Six rats in the control group, as the $0 \mathrm{~h}$ samples in sham-operated groups and partial hepatectomy groups, received the perfusion immediately after the surgical removal of left and median lobes.

\section{Isolation of pit cells}

After the rats were anesthetized with ether and sterilized with $75 \%$ alcohol, the abdominal cavity was opened to expose the portal vein, and the renal and inferior vena cava were ligated after hepatic portal vein catheterization. Liver perfusion was carried out by a two-step perfusion method. Then, the perfused livers from six rats were collected together and cut into pieces and digested with $15 \mathrm{~mL} 0.05 \%$ IV-type collagenase at $37^{\circ} \mathrm{C}$ for $15 \mathrm{~min}$. The digested mixed cell suspensions were filtered through a 400-mesh net, and the filtered solution was centrifuged three times for $3 \mathrm{~min}$. The resulting precipitate was harvested and washed twice using PBS at $4^{\circ} \mathrm{C}$. Cell concentration was adjusted to $1 \times 10^{8}$ cells $/ \mathrm{mL}$. Six milliliters of the mixed cell suspension was layered on the surface of $4 \mathrm{~mL} 60 \%$ Percoll (Pharmacia), followed by centrifugation at $200 \mathrm{~g}$ at $4^{\circ} \mathrm{C}$ for $5 \mathrm{~min}$. The resulting supernatant was mixed with an equal volume of PBS and centrifuged at $400 \mathrm{~g}$ for $2 \times 2 \mathrm{~min}$, and the sediment at the lower layer was the high-density nonparenchymal cell fraction containing pit cells. After cell concentrations were adjusted to $1 \times 10^{8}$ cells $/ \mathrm{mL}$, the pit cell-enriched solution was incubated with $10 \mu \mathrm{L} / \mathrm{mL}$ rat anti-CD161a antibody for $15 \mathrm{~min}$ at $4^{\circ} \mathrm{C}$, and then mixed with 10 $\mu \mathrm{L} / \mathrm{mL}$ rat anti-PE magnetic beads for another $15 \mathrm{~min}$ at $4^{\circ} \mathrm{C}$. The cell suspension was loaded onto the separation column and allowed to flow naturally. PBS was passed through the column and fractions were collected, and the collected solutions were the suspension for pit cells (He et al., 2009).

\section{Immunochemical analysis}

A few mixed cell suspension and purified pit cells were individually fixed with $10 \%$ formaldehyde for $30 \mathrm{~min}$, and then smeared onto glass slides. When cell suspensions on glass slides dried, microwave antigen retrieval was done. The sections were incubated separately with a 1:2000 dilution (v/v) of CD8 and CD56 antibodies overnight at $4^{\circ} \mathrm{C}$, then the 1:5000 (v/v) diluted biotin-labeled secondary antibody was incubated for $60 \mathrm{~min}$ at $37^{\circ} \mathrm{C}$. The system was hybridized with streptavidin-biotin complex at $37^{\circ} \mathrm{C}$ for $30 \mathrm{~min}$. Meanwhile, the paraffinized sections of liver tissue at the corresponding times were prepared for immunohistochemical staining. The results were observed and analyzed under an optical microscope.

\section{Microarray analysis}

Total RNAs were prepared from pit cells of 6 rats per group according to the manual of the Trizol reagent (Invitrogen Corporation, Carlsbad, CA, USA) (Norton, 1992). RNA quality was assessed by agarose electrophoresis and spectrophotometric analysis prior to cDNA synthesis (Scott, 1995). The amplification and biotinylation of probes were done for 
microarray analysis according to Affymetrix recommendations. Samples were hybridized to the Rat Genome 2302.0 Array produced by Affymetrix Inc. (Santa Clara, CA, USA). Washing and scanning of the GeneChips were according to the manufacturer protocol. The detection and quantitation of the hybridization were done using GeneChip scanner 3000 (Affymetrix Inc.) (Kube et al., 2007). All arrays were assessed for 'array performance' prior to data analysis. Control transcripts were spiked into the hybridization mixture to control the hybridization efficiency and sensitivity. To minimize potential systematic errors, all stages of the experiment were balanced across experimental groups. That is, an equal animal number in every group was sacrificed within the same time, and the equal RNA preparations from the representative groups were processed through the labeling, hybridization, washing, and scanning protocols at a given time.

\section{Data analysis and normalization}

The data for each microarray were initially normalized by scaling all signals to a target intensity of 200. Expression analysis was performed using the defaults parameter settings. Present calls required $\mathrm{P}<0.05$, and marginal calls required $0.05<\mathrm{P}<0.065$; probe sets with $\mathrm{P}>0.065$ were marked absent. Each array was analyzed based on the present (gene expression), marginal (marginal expression), or absent (no expression) call for each probe set. Gene's relative values were evaluated by the ratio of the normalized signal value of experimental groups (including SO groups and partial hepatectomy groups) at different times to that of the control group, e.g., a gene with $\geq 3$ relative value was regarded as an up-regulated gene; a gene with $\leq 0.33$ relative value, as a down-regulated gene; a gene with $0.33 \sim 2.99$ relative value, as insignificantly expressed. To minimize the technical error from array analysis, the cell samples at each time were repeated thrice with a chip. The average relative value of three repeated analyses at each time was calculated as effective values. Finally, these values were analyzed with GeneMath, GeneSpring (Silicon Genetics, San Carlos, CA, USA) (Wang et al., 2007; Guo et al., 2008).

\section{RT-PCR}

To assess the array results, real-time polymerase chain reaction (RT-PCR) was performed. RNA samples for RT-PCR were from the isolated pit cells of the cell samples corresponding to different times. Double-stranded cDNA template preparation and purification were performed with a reverse transcription kit (Promega) according to the manufacturer protocol. The primer sequences were obtained for six genes, including $b 2 m, k l r b 1 a$, ncam l, $h k 1$, trim 24 , and $u b c$, and synthesized following the protocol described by Wang and Xu (2010). Amplification of target cDNA and the standard curve construction were also in accordance with the method of Wang and $\mathrm{Xu}(2010)$.

\section{Gene functional annotation and statistical analysis}

The normalized signal ratio in the chip analysis was log2-transformed for classifying the patterns of serial changes of gene expression. The Gene Ontology Database (www. geneontology.org/) was used as a standard source for gene annotation information. To examine statistical significance for frequencies of genes of each functional group in each cluster, we used the modified Fisher exact test to measure the gene set enrichment in the annotation terms. 


\section{RESULTS}

\section{Changes in morphology and number of pit cells during liver regeneration}

Based on immunohistochemical staining, we observed that in the normal control group CD8- and CD56-positive pit cells surrounded the hepatic sinusoid. As the regeneration went on, the number of positive pit cells gradually increased; they began to decline $36 \mathrm{~h}$ after partial hepatectomy. Up to $72 \mathrm{~h}$, the number of positive pit cells gradually returned to control levels (Figure 1A and B). Immunocytochemistry analysis showed that most of the pit cells from the control group exhibited the irregular cell morphology, CD8- and CD56-positive staining of the cytoplasm, and prominent nucleoli with high refractivity (Figure 1C, D). As the regeneration progressed, pit cells became bigger and rounder, and showed a slightly atypical shape, accompanied by weakened cytoplasm staining. After $72 \mathrm{~h}$, the staining became lighter in the cytoplasmic region around the nucleus. By $168 \mathrm{~h}$, pit cells were similar to normal in cell morphology.

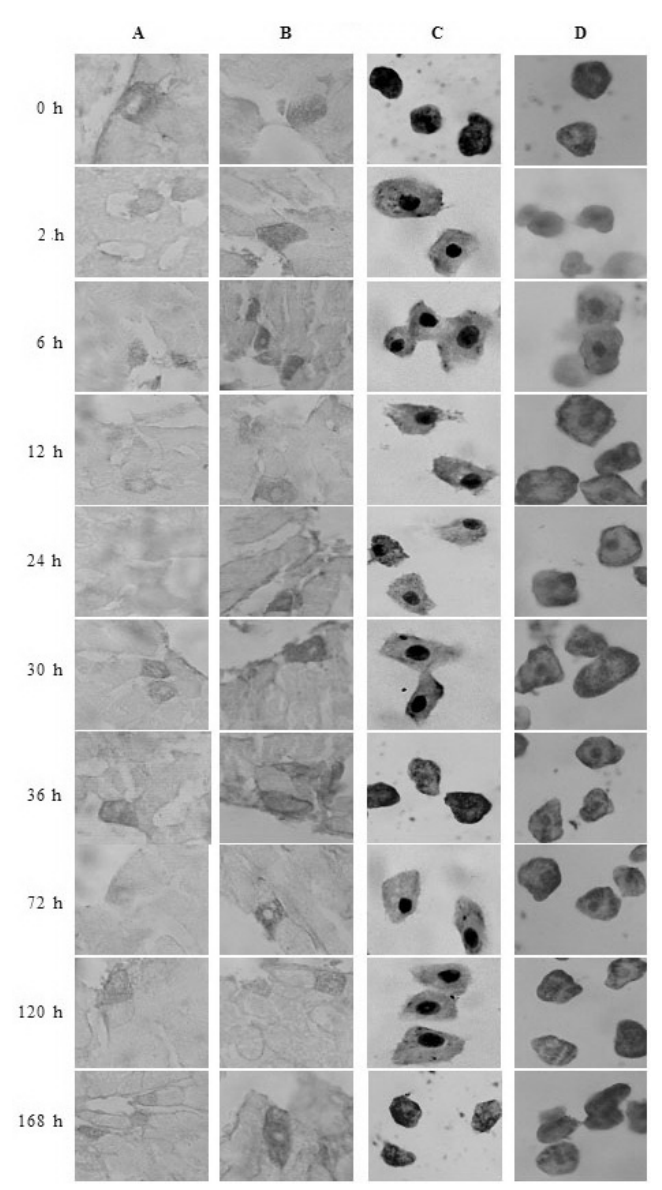

Figure 1. Immunochemical images of CD8- and CD56-labeled liver tissues and pit cells at different times after partial hepatectomy (40X). A. and B. Immunohistochemical CD8 and CD56 staining for the regenerating liver, respectively. C. and D. Immunocytochemical CD8 and CD56 staining for pit cells, respectively. 


\section{The yield, purity and survival rate of pit cells}

Based on the methods of two-step perfusion, collagenase digestion and Percoll density gradient centrifugation, we applied magnetic beads isolation method to attain high yield and vitality of pit cells from the regenerating rat liver. The average number of pit cells per rat from different recovery times is shown in Figure 2A. The survival rate of the purified pit cells was at least $96 \%$ (Figure 2B). Immunocytochemistry-based statistical analysis showed that CD8- and CD56-positive pit cells sequentially occupied at least 95.05 and $95.02 \%$ of the isolated pit cells, respectively (Figure 2C).
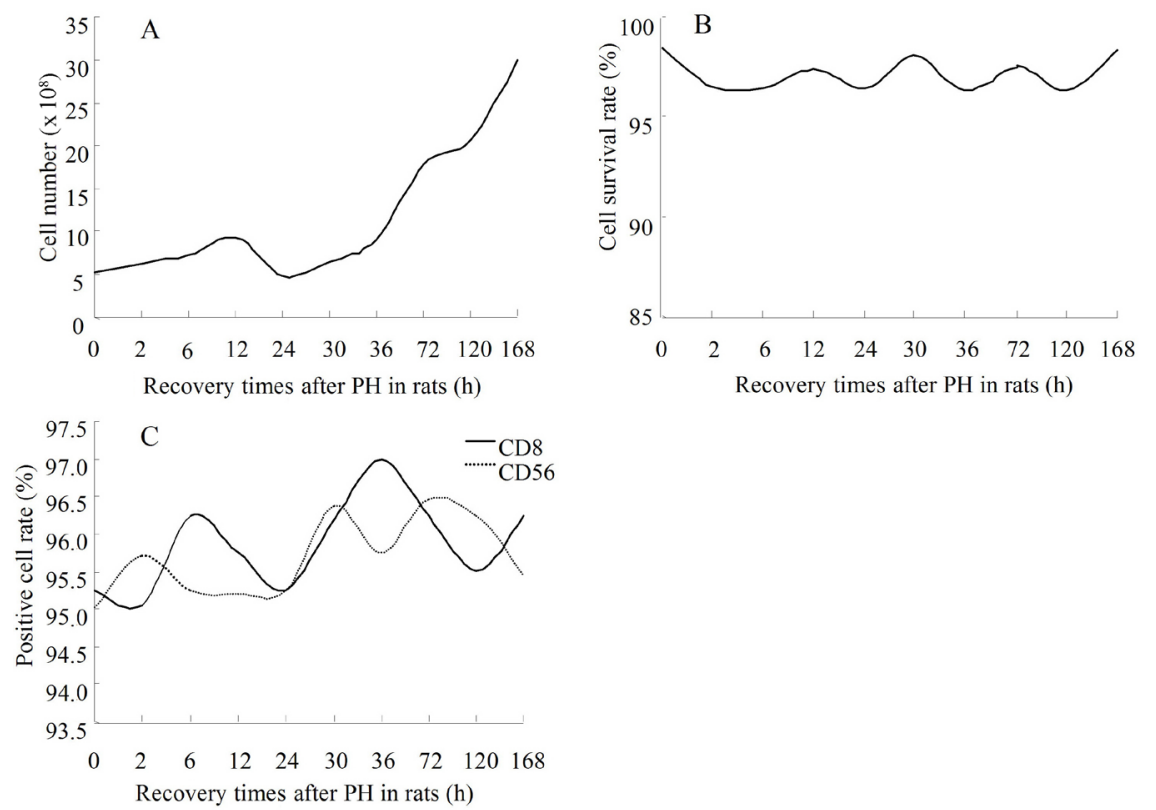

Figure 2. Isolation and purification of rat pit cells. A. Mean number of pit cells obtained from each rat. B. Cell survival rate after purification. $\mathbf{C}$. The positive cell rate in the isolated pit cells. $\mathrm{PH}=$ partial hepatectomy.

\section{Identification of differentially expressed genes of pit cells from regenerating rat liver}

We analyzed the global gene expressions of pit cells from the regenerating rat liver at different times after partial hepatectomy. Of 25,020 genes in the Rat Genome 2302.0 Array, 970 genes, composed of 612 known and 358 unknown genes, were significantly differentially $(\mathrm{P} \leq 0.05 ; t$-test) expressed in partial hepatectomy groups compared with the control group, had the same or similar expression levels in three chip analyses and a 3 -fold change or above in expression level at least one time. These genes were identified as liver regeneration-related genes. Figure 3 illustrates the number of up- or down-regulated genes at each time after partial hepatectomy. According to the gene expression changes during liver regeneration, even a short period of partial hepatectomy treatment $(0.5-2 \mathrm{~h})$ can induce robust changes in cellular mRNA pool and activate a large number of genes (Figure 3). As the regeneration went on, there was 
an increase in the number of differently expressed genes. The down-regulated genes were more frequent than the up-regulated genes from 6-12 $\mathrm{h}$ after partial hepatectomy; the contrary occurred from 24-36 h. The down-regulated genes were predominant at other times. By $168 \mathrm{~h}$, the number of regulated genes decreased, suggesting completion of liver regeneration.

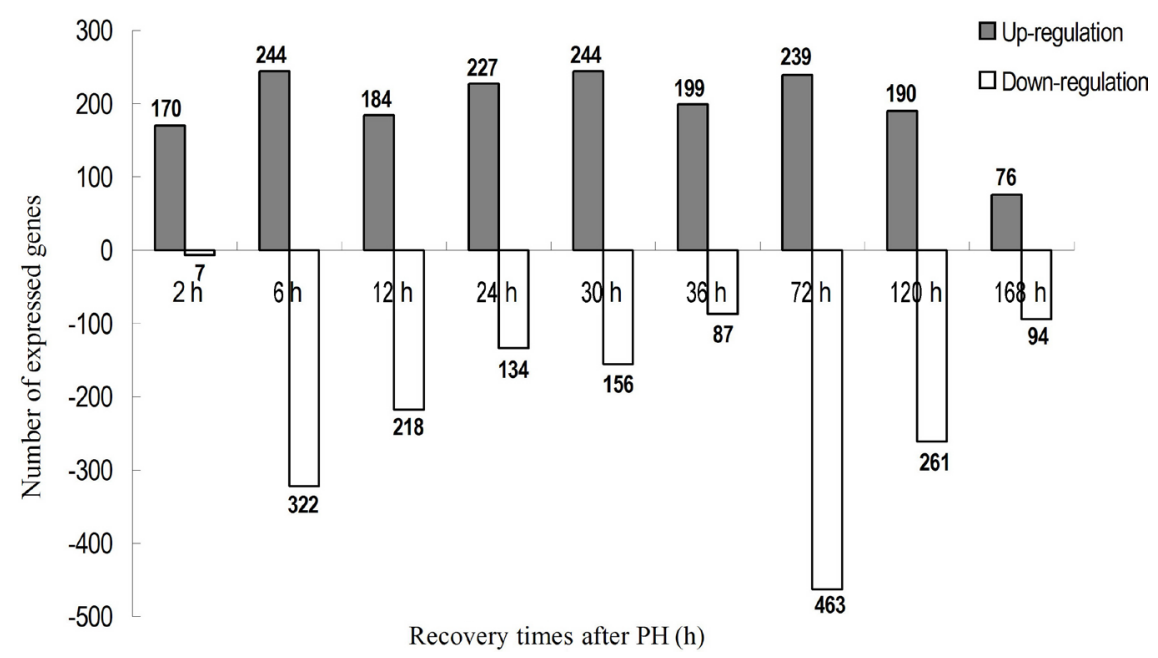

Figure 3. The number of genes with significant fold changes $(\geq 3)$ is shown at the indicated times. Gray and white columns represent the number of up- and down-regulated genes at each time, respectively. $\mathrm{PH}=$ partial hepatectomy.

\section{Validation of chip results by RT-PCR}

RNAs from 10 pit cell samples from regenerating rat liver were used for microarray analysis. The expression changes of six selected genes, $b 2 m$ (beta-2 microglobulin), klrbla (ller cell lectin-like receptor subfamily B member 1A), ncam 1 (neural cell adhesion molecule 1), $h k l$ (hexokinase 1), trim 24 (tripartite motif-containing 24), and $u b c$ (ubiquitin C) by microarray were identified by RT-PCR assay. RT-PCR results showed that the mRNA level of $u b c$ was different from that from the chip data, but the expression tendency was similar by both methods. Fold change of other five genes detected by RT-PCR almost correlated with the direction of fold change obtained from chip data, confirming the validity of the microarray gene expressions. Standard deviations for the six genes were calculated based on three repeated chip analyses of pit cell samples at each time (Figure 4).

\section{Gene expression profiles in pit cells from regenerating rat liver}

When compared with the control (pit cell samples from untreated rats), the gene expression profiles were significantly up- or down-regulated in the experimental groups (pit cell samples from partial hepatectomy-treated rats). In total, 612 known genes were detected to be differentially expressed in the experimental groups. To understand the molecular mechanism of the role of pit cells in partial hepatectomy-treated cells, hierarchical clustering algorithm was used to group genes based on the similarity of expression patterns. Each row in Figure 


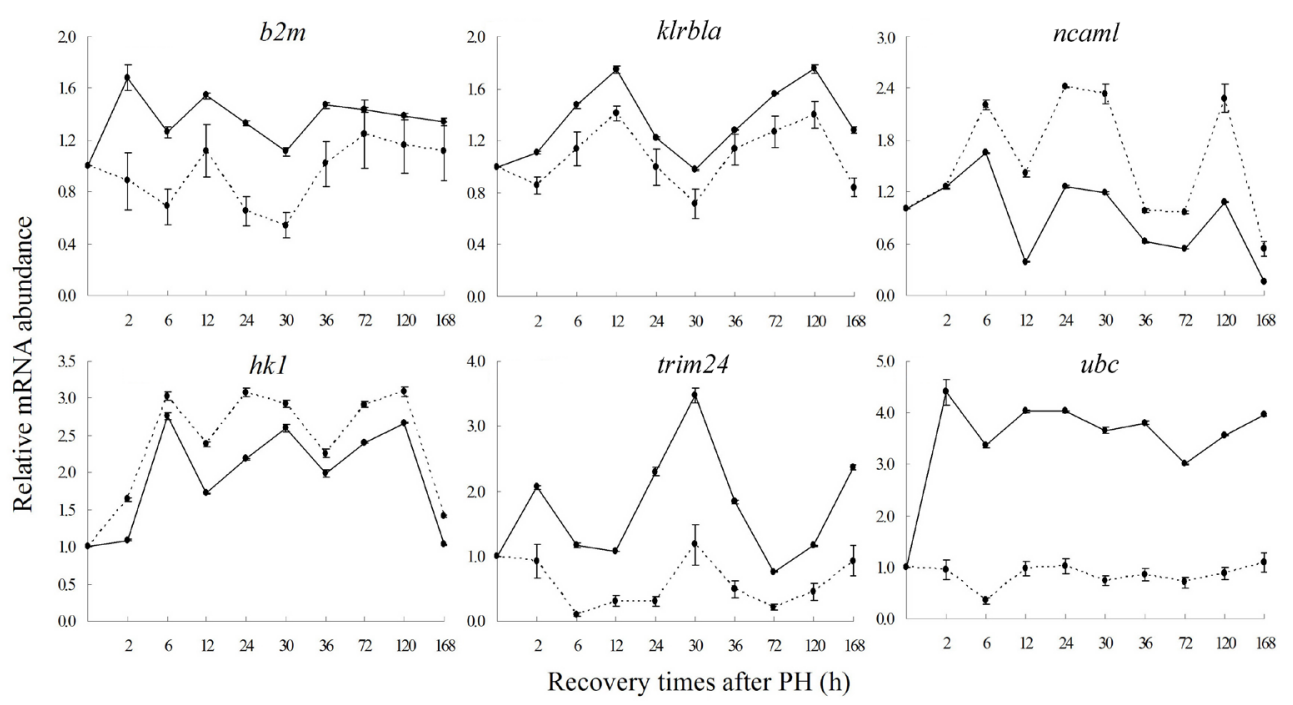

Figure 4. RT-PCR validation of six genes $b 2 m$, klrbla, ncaml, $h k 1$, trim 24 , and $u b c$. The results of RT-PCR are shown by solid lines; the results of array are shown by dotted lines. The times are 2, 6, 12, 24, 30, 36, 72, 120, and $168 \mathrm{~h}$ after partial hepatectomy (PH). Standard deviation of the six genes from array data was added, which confirms the reliability of the chip data.

4 represents the expression changes of each gene after partial hepatectomy, and each column represents the expression levels of all genes in a single chip. The expression level of each gene was visualized in color, relative to its median expression level compared with that of the control. Red and green colors denote expression levels higher and lower than the control, respectively, and the color intensity denotes the degree of deviation from the control. Six hundred and twelve known genes exhibited two distinct expression patterns: up-regulation and down-regulation. There were 204 up-regulated and 408 down-regulated genes. The 612 genes were functionally categorized based on the Gene Ontology database.

\section{Functional analysis of the pit cell transcriptome}

The 204 up-regulated and 408 down-regulated genes in pit cells were grouped into at least 23 functional categories (Figure 5), such as signal transduction, metabolism, cellular organization and biogenesis, cell growth, proliferation, growth, differentiation, cell adhesion, immunity, inflammation, phagocytosis, secretion of active substance, and so on. Gene enrichment function analysis revealed that the genes encoding proteins for the categories "signal transduction", "cell proliferation", "immune response", "cell differentiation", "cell adhesion, "secretion of active substance", "cell chemotaxis", "cell migration", "inflammatory response", and "nucleic acid metabolism" were significantly enriched in an up-regulation pattern, involving $23,33,27,34,19,8,9,8,17$, and 10 genes, which in order accounted for $11.3,16.2,13.2,16.7,9.3,3.9,4.4,3.9,8.3$, and $4.9 \%$ of the 204 up-regulated genes. Similarly, the genes encoding proteins for carbohydrate metabolism, lipid metabolism, amino acid metabolism, detoxification, and secondary metabolism were frequently distributed in a down-regulation pattern, involving $20,57,36,42$, and 31 genes, which separately accounted for 4.9, 14.0, 8.8, 10.3, and 7.6\% of 408 down-regulated genes (Table 1). 


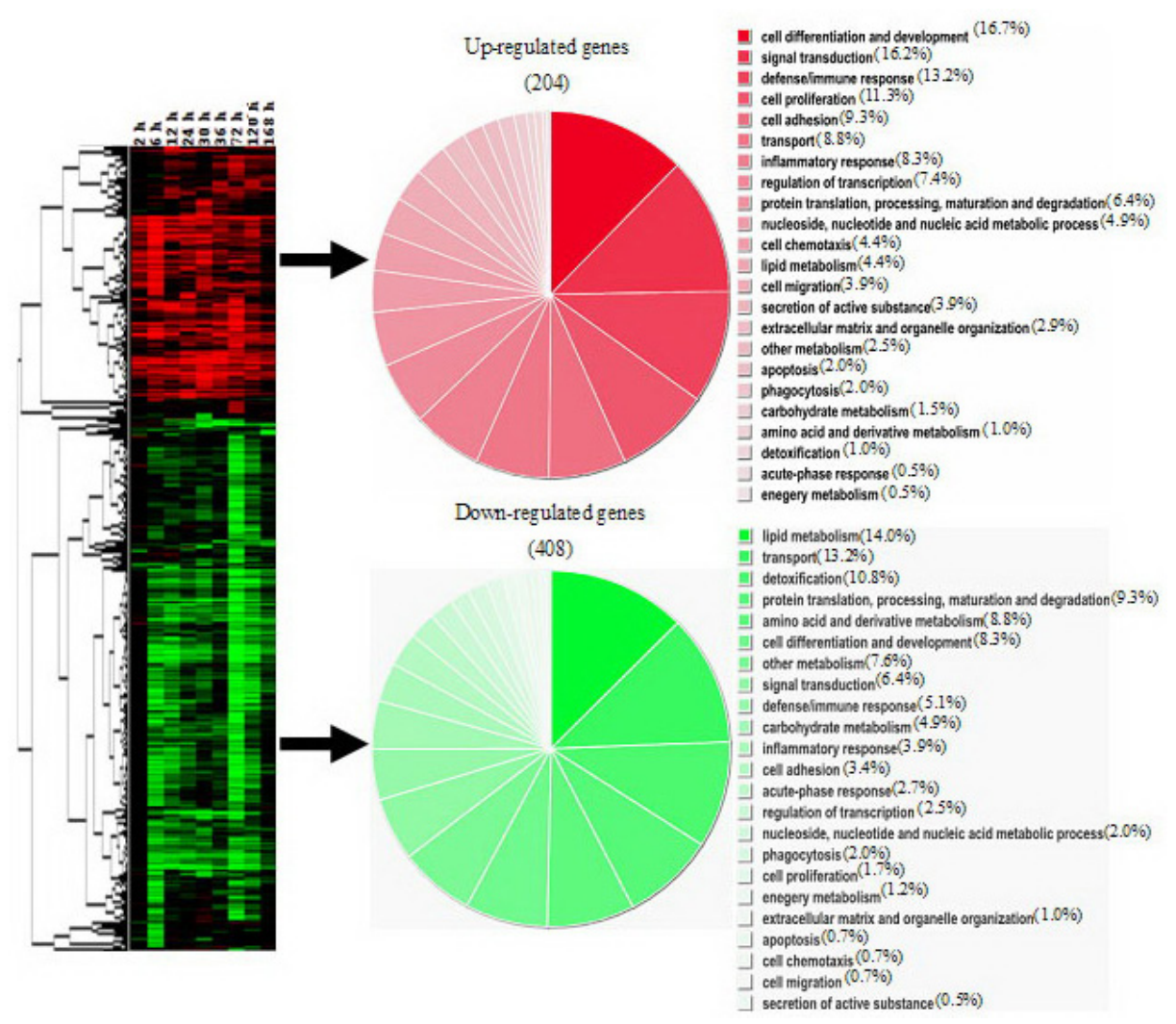

Figure 5. Clustergram of up- and down-regulated genes in pit cells during liver regeneration. Each gene is represented by a single row of clustered boxes; each experimental cell sample is represented by a single column. The entire clustered image is shown on the left. The functional categories of up- and down-regulated genes are shown in the right panel.

Table 1. Significantly enriched gene function categories in up- and down-regulation patterns based on gene expressions of pit cells from regenerating rat liver.

\begin{tabular}{lcc}
\hline Biological pathways & Gene counts & P \\
\hline Up-regulated pathway & & \\
Cell proliferation & 23 & 0.0000 \\
Signal transduction & 33 & 0.0001 \\
Defense/immune response & 27 & 0.0006 \\
Cell differentiation & 34 & 0.0019 \\
Cell adhesion/migration & 19 & 0.0028 \\
Secretion of active substance & 8 & 0.0032 \\
Cell chemotaxis & 9 & 0.0035 \\
Inflammatory response & 17 & 0.0205 \\
Nucleic acid metabolism & 10 & 0.0410 \\
Down-regulated pathway & & \\
Detoxification & 42 & 0.0000 \\
Amino acid and derivative metabolism & 36 & 0.0000 \\
Lipid metabolism & 57 & 0.0001 \\
Secondary metabolism & 31 & 0.0063 \\
Carbohydrate metabolism & 20 & 0.0244 \\
\hline Ge
\end{tabular}

Gene counts $=$ the number of differently expressed genes in different categories; $\mathrm{P}=$ significant ontological classification evaluated by the Fisher exact test. 


\section{Gene functional categories significantly enriched in an up-regulation pattern}

According to chip data, pit cells markedly up-regulated the following functional classified genes: 33 genes related to signal transduction pathways including TGF- $\beta$-mediated signal pathway (aspn, ltbp4, etc.), small GTPase-mediated signal pathway (arl11, rhoq, etc.), neuropeptidemediated signal pathway (oprdl, $n m b$, etc.), G-protein coupled receptor protein signaling pathway (htrld, prlhr, etc.), and cytokine and chemokine-mediated signal pathway (ccl2, lilrb3, etc.) at 6 $\mathrm{h}$ post-partial hepatectomy. Twenty-six cell proliferation-related genes had increased expression at 24-72 $\mathrm{h}$ after partial hepatectomy, including cyclin-dependent kinase genes $c d c a 2, c d c 25 b$, etc., kinesin family member genes kifcl, kif2c, etc., spindle and centromere behavior-related genes bub1, ckap2, nuf2, ndc80, and spc24, transcription factor genes $\operatorname{tnf,~fabp4,~pdpn,~etc.,~and~DNA~}$ metabolism genes (DNA polymerase components polk, poln, etc., and DNA repair genes fancd2, fen1, lig1, etc.). Twenty-seven cell adhesion/migration genes were up-regulated at 6-72 $\mathrm{h}$ after partial hepatectomy, mainly encoding extracellular matrix components ( $p c d h 19, p d p n, p k p 1$, podxl2, spp1, etc.), lectins (clec12a, clec7a, etc.), gap junction proteins (gja5, gjb6, etc.), and cell migration-related proteins ( $c c l 2, c d 44$, corola, cxcr4, mmp9, pdpn, tnf, vcan, etc.). Thirty-four cell differentiation-involved genes, showing the up-regulation trends during the whole liver regeneration, mainly contained cytokine genes $f g f 14$, $\operatorname{tnf}$, etc., transcription factor genes trp53, zeb2, etc., and extracellular matrix components timpl, vcan, etc. Nine genes for cell chemotaxis, such as $c c l 2$, $c x c l 2, i l 1 b$, and $i l 8 r b$, were enhanced in expression mainly at $72 \mathrm{~h}$ after partial hepatectomy. Genes involved in active substance secretion, mostly encoding the components of chemokines and cytokine-mediated signal pathways, i.e., tnf, ill $r n$, and $p t p n 11$, were induced during early phase (2-6 h post-partial hepatectomy) of liver regeneration. Twenty-seven immune/defense response genes and 17 inflammatory response genes, had increased mRNA abundance, especially at $72 \mathrm{~h}$ post-partial hepatectomy; they mainly code for chemokines $c c l 2, c x c l 2$, etc., interleukins $i l 1 b$, illr2, etc., and MHC-I molecules $r t 1 a-2$, and $r t 1-a 3$, etc. (Figure 6).

\section{Gene functional categories significantly enriched in down-regulation patterns}

A large number of cellular metabolism-involved genes were down-regulated by pit cells (Table 1). Of 20 carbohydrate metabolism genes, 14 genes for carbohydrate synthesis, including gluconeogenesis ( $a t f 3, f b p 1, g 6 p c, g p d 1, p c k 1$, tat, gpt, etc.) and carbohydrate synthesis (gys2, shpk, etc.), were initially decreased at $6 \mathrm{~h}$ after partial hepatectomy, with minimum levels at $72 \mathrm{~h}$. Among lipid metabolism-related genes, 23 lipid synthesis genes, encoding proteins for sterol synthesis (cyp17al, dhcr 7, hmgcs 2, hsd17b11, apoal, etc.), fatty acid synthesis (acsbgl, acot 12, apoa5, fads2, sc 5d, scd1, etc.), glycerol synthesis (ang1, apoh, etc.), and phospholipid synthesis (etnk2, pemt, etc.), were reduced mainly at $6 \mathrm{~h}$ after partial hepatectomy. Twentyone lipid catabolism-related genes showed decreased mRNA levels at $72 \mathrm{~h}$, mainly encoding the proteins for fatty acid oxidation ( $h a c l 1$, acox2, decrl, acadm, adh7, etc.), triglyceride catabolism (apob, apoc3, cps1, etc.) and lipid degradation (enpp2, lipc, angptl3, etc.). Amino acid metabolism genes were significantly decreased at $72 \mathrm{~h}$ after partial hepatectomy; they are mainly involved in metabolism of amino acids such as arginine ( $\operatorname{ass} 1, \arg 1$, etc.), alanine (dpys, upb1, etc.), cysteine (cth, cbs, etc.), glutamine ( $g l s 2, c p s 1$, etc.), glycine ( $s a r d h, d m g d h$, etc.), phenylalanine ( $h p d, p a h$, etc.), methionine (bhmt, bhmt2, etc.), and tryptophan (kynu, tdo2, etc.), as well as amino acid-derivative metabolism. The mRNA levels of 42 genes in the cat- 


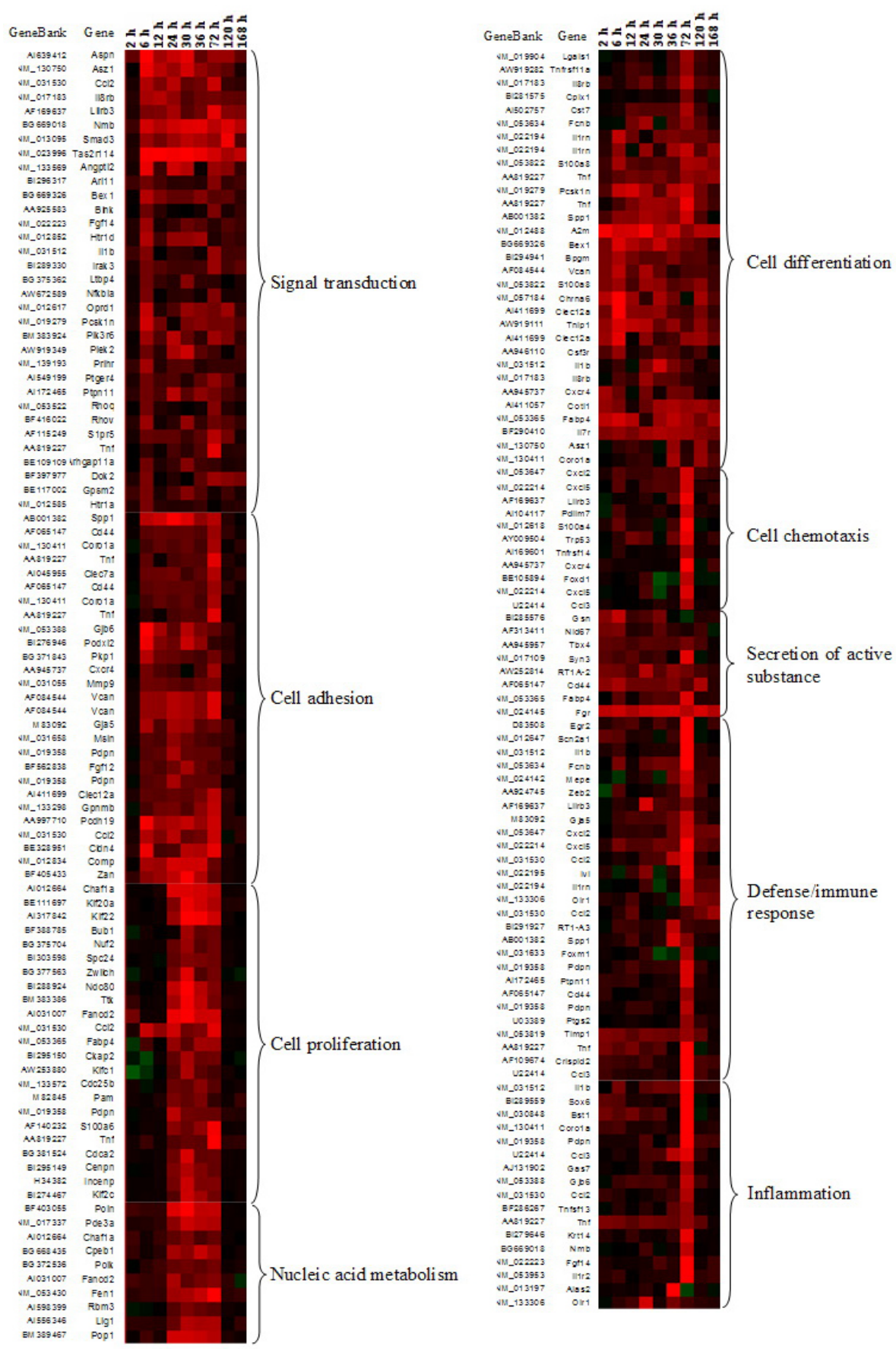

Figure 6. Clustering of the biological activities significantly enriched in up-regulation pattern. Red and black colors signify up-regulation and insignificant expression, respectively. 
egory "detoxification" were observed to be markedly below control between 6-72 $\mathrm{h}$ after partial hepatectomy; they mainly encoded products for drug metabolism, such as cytochrome p450 family genes cyp2a2, cyp $2 \mathrm{b3}$, monooxygenase genes fmol, fmo3, fmo5, and sulfotransferase genes sult1a1, sult1c1, for alcohol metabolism (akr1c12, aldh1a1, cyp2f4), for glutathione metabolism, such as glutathione transferase genes, gstm 1, gstt1, gsta3, and for other metabolism xenobiotic substances, such as cytochrome p450 family genes (Figure 7).
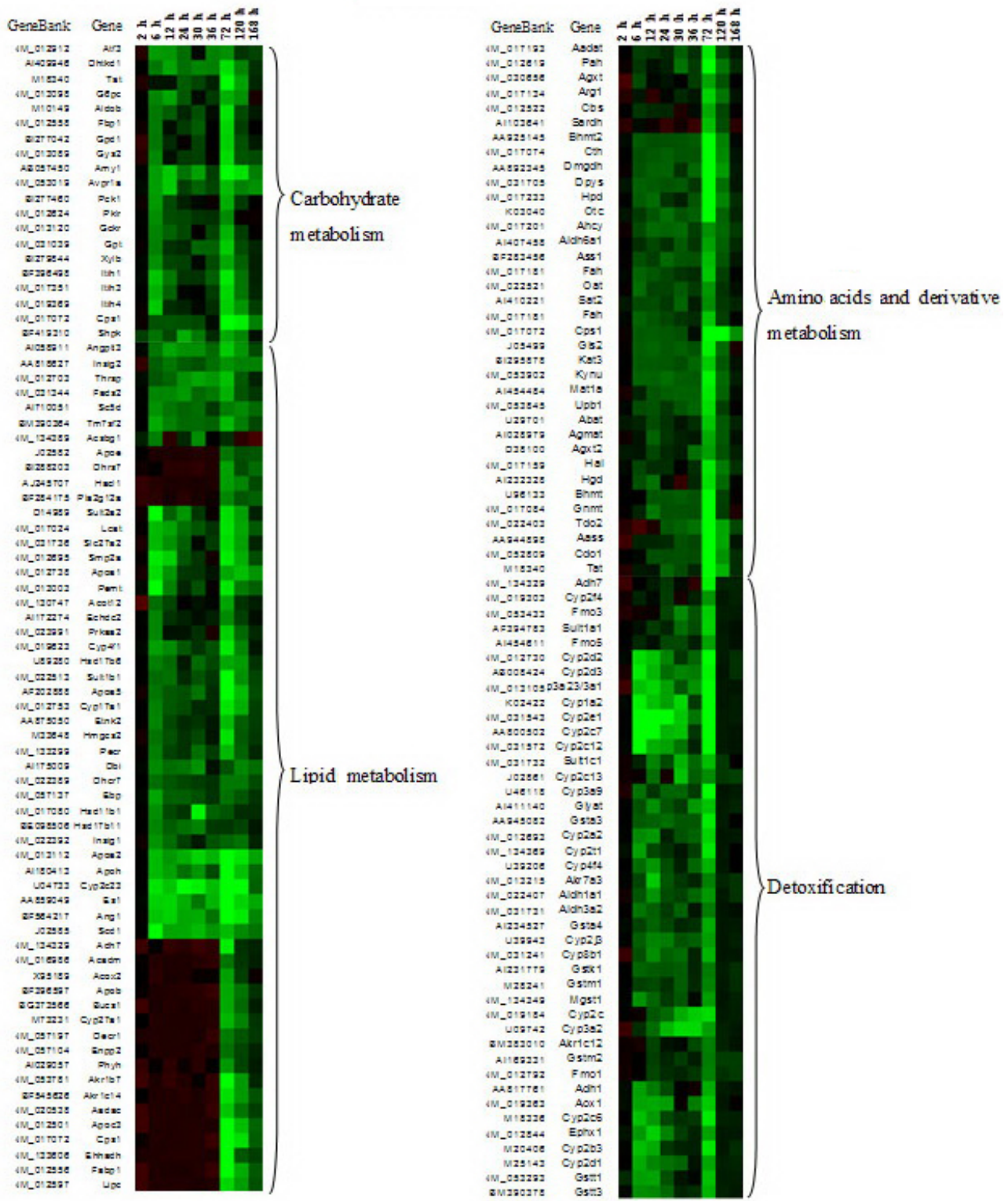

Figure 7. Clustering of biological activities significantly enriched in down-regulation pattern. Green and black colors signify up-regulation and insignificant expression, respectively. 


\section{DISCUSSION}

Pit cells, a hepatic intrasinusoidal population of large granular lymphocytes (Bouwens and Wisse, 1992), have been recently considered to play a crucial role in liver regeneration. We made a genome-wide analysis of the relationship between pit cells and liver regeneration. We first isolated pit cells of high purity $(>96 \%)$ from regenerating rat liver, then examined the temporal gene expression profiles of pit cells. We identified 612 known genes and 358 unknown genes as liver regeneration-related genes. The 612 known genes were grouped into up-regulation and down-regulation patterns, and were found to be involved in about 23 major biological activities, according to gene ontology analysis. Gene functional enrichment analysis indicated that 10 functional categories, including signal transduction and cell proliferation, were significantly upregulated pattern, while the categories "cellular metabolisms" were down-regulated.

Studies have demonstrated that many genes involved in cytokine networks are stimulated after partial hepatectomy, subsequently causing changes in various biological activities in pit cells by triggering growth factor- and cytokine-mediated pathways (Dong et al., 2007). According to microarray chip data obtained from our study, the cytokine secretion-regulatory genes and the cytokine-mediated signal pathway genes begin to have increased mRNA production at an early phase. Consistently, we observed that a substantial number of genes encoding cytokines and growth factors, such as $t h f, c c l 2, c c l 3, c x c l 2, c x c l 5, f g f 12$, and $f g f 14$, had elevated expression at 2-6 h after partial hepatectomy. Zheng et al. (2009) reported that pit cells could up-regulate many cytokines (i.e., TNF- $\alpha$ ) after partial hepatectomy. Furthermore, these cytokines can activate downstream signaling cascades (Ravandi et al., 2003). Some genes, such as cytokine and chemokine-mediated signal pathway genes lilrb3, irak3, TGF- $\beta$ signaling pathway genes aspn and ltbp 4, and neuropeptide signaling pathway genes oprd 1 and $n m b$, were up-expressed at $6 \mathrm{~h}$ after partial hepatectomy in our study. Diehl and Rai (1996) also pointed out the early up-regulation of genes in the signal pathways after partial hepatectomy, postulating the implication of these pathways in the initiation of liver regeneration. According to the above analysis, cytokine and growth factor-mediated signal pathways in pit cells are enhanced at an early phase of liver regeneration.

After hepatic mass loss in rats, there is a large variation between different liver cell types with regard to the onset and peak of cell replication (Clavien, 2008). For instance, the peak for hepatocyte proliferation is at $24 \mathrm{~h}$ post-partial hepatectomy, for cholangiocytes at $48 \mathrm{~h}$, Kupffer cells at $72 \mathrm{~h}$, and sinusoidal endothelial cells at $96 \mathrm{~h}$. Although the onset time and peak time for pit cell replication have not yet been reported, we observed that cell proliferation-related genes are significantly up-regulated pattern in pit cells from $24-72 \mathrm{~h}$ after partial hepatectomy.

One of the key events occurring during cell cycle progress is DNA replication (Taub, 2004). Ten DNA metabolism-involved genes, mainly related to DNA synthesis and DNA repair, were also found to be up-regulated 24-72 $\mathrm{h}$ after partial hepatectomy, which coincided with pit cell replication, thus providing cell division with ample materials. Based on our analysis, we suggest that the self-replication of pit cells is largely responsible for the compensation of cell numbers during liver regeneration.

Cell adhesion plays an important role in many biological processes, such as intercellular communication, cell differentiation, and so on (Zheng et al., 2009). Revel et al. (1980) investigated intercellular communication in the regenerating rat liver, observing a drastic reduction in gap junctions at 29-35 h after partial hepatectomy. Previously, we had found that many gap 
junction-related genes ( $g j a 7, \operatorname{tg} f b 3$, etc.) are enhanced during the early period of liver regeneration ( $\mathrm{Li}$ et al., 2007). Here, we found a significant distribution of 19 cell adhesion genes to be up-regulated in pit cells. These genes were up-regulated 6-72 h after partial hepatectomy, mainly consisting of extracellular matrix component genes $p c d h 19$, pdpn, etc., lectin family genes $\operatorname{clec} 12 a$, clec $7 a$, and gap junction membrane channel protein family genes gja5, gjb6, etc. This implies that the increased expression of cell adhesion-related genes might be linked to the attenuated adhesion of pit cells after partial hepatectomy. Also, cell migration genes frequently present in the same pattern as cell adhesion genes, such as corola, mmp 9, pdpn, and vcan, also showed enhanced expression at 6-72 $\mathrm{h}$ following partial hepatectomy, which might be attributed to the reduced cell adhesion assumed above. The interrelationship between cell adhesion and cell migration is necessary for cell differentiation and development. Our micro-array chip results showed that a larger proportion of cell differentiation-related genes, including genes encoding cytokines, transcription factors and extracellular matrix components, were up-regulated at different regeneration phases; this demonstrated that differentiation machinery for pit cells commenced at the onset of liver regeneration and at the termination phase of liver regeneration.

As one of the important cellular components constituting the hepatic immune system, pit cells can produce many inflammatory factors and chemokines, modulating the inflammation and immunity of injured liver (Gao et al., 2009). In our study, pit cells began to strongly induce the expression of chemotaxis-related genes at $72 \mathrm{~h}$ after partial hepatectomy, such as chemokine genes $c c l 2$ and $c x c l 2$, and interleukin genes $i l l b$ and $i l 8 r b$. Our previous studies on the regenerating rat liver showed that the expressions of the above genes were induced early post-partial hepatectomy (Xu et al., 2009). Gene function enrichment analysis indicated that immune/inflammatory response genes had increased transcript levels at $72 \mathrm{~h}$, including genes encoding proinflammatory factors, chemokines, interleukins, MHC molecules, and secretory proteins, while the tissue-level study performed by Wang et al. (2009) showed that some inflammatory factor genes thf, procr, proc, thbd in the regenerating rat liver were increasingly expressed at $2 \mathrm{~h}$ after partial hepatectomy. The study of Su et al. (2002) regarding the gene expression at priming phase of liver regeneration in mice found that inflammatory genes il6, $c d 14, m c p 1$, gro were highly induced at early stages of liver regeneration. According to the above analysis, we conclude that the high expressions of the above genes at $72 \mathrm{~h}$ in pit cells may imply production and activity of inflammatory factors at the later phase of liver regeneration, which is inconsistent with previous observations at the tissue level, requiring an in-depth investigation to explain this disagreement.

The genes for the category "cell metabolism" were significantly frequent in a downregulation pattern, including genes involving metabolism of carbohydrates, lipids, amino acids, drugs, toxins, and other xenobiotics. We detected down-regulation of these genes at 6-72 $\mathrm{h}$ after partial hepatectomy, which suggested a repressed cellular metabolism in pit cells after liver regeneration. However, these findings are from micro-array chip data. The chip in this study can only be used to measure gene transcriptional profiles; it cannot quantify the translation process, let alone reflect the true biological process in pit cells. We plan to test these hypotheses using methods such as gene addition, interference RNA and protein-protein interaction.

\section{ACKNOWLEDGMENTS}

Research supported by the National Basic Research 973 Pre-Research Program of China (\#2010CB534905). 


\section{REFERENCES}

Bouwens L and Wisse E (1992). Pit cells in the liver. Liver 12: 3-9.

Clavien PA (2008). Liver regeneration: a spotlight on the novel role of platelets and serotonin. Swiss Med. Wkly. 138: 361-370.

Diehl AM and Rai R (1996). Review: regulation of liver regeneration by pro-inflammatory cytokines. J. Gastroenterol. Hepatol. 11: 466-470.

Dong Z, Wei H, Sun R and Tian Z (2007). The roles of innate immune cells in liver injury and regeneration. Cell Mol. Immunol. 4: 241-252.

Francavilla A, Vujanovic NL, Polimeno L, Azzarone A, et al. (1997). The in vivo effect of hepatotrophic factors augmenter of liver regeneration, hepatocyte growth factor, and insulin-like growth factor-II on liver natural killer cell functions. Hepatology 25: 411-415.

Gao B, Radaeva S and Park O (2009). Liver natural killer and natural killer T cells: immunobiology and emerging roles in liver diseases. J. Leukoc. Biol. 86: 513-528.

Griffini P, Smorenburg SM, Vogels IM, Tigchelaar W, et al. (1996). Kupffer cells and pit cells are not effective in the defense against experimentally induced colon carcinoma metastasis in rat liver. Clin. Exp. Metastasis 14: 367-380.

Guo W, Cai C, Wang C, Zhao L, et al. (2008). A preliminary analysis of genome structure and composition in Gossypium hirsutum. B. M. C. Genomics 9: 314.

He CX, Lai XF, Wang L, Jin YF, et al. (2009). Isolation, purification and identification of pit cells in rat liver. Henan Sci. 27: $1072-1076$.

Higgins GM and Anderson RM (1931). Experimental pathology of the liver I. Restoration of the liver of the white rat following partial surgical removal. Arch. Pathol. 12: 186-202.

Kmiec Z (2001). Cooperation of liver cells in health and disease. Adv. Anat. Embryol. Cell Biol. 161: III-151.

Kube DM, Savci-Heijink CD, Lamblin AF, Kosari F, et al. (2007). Optimization of laser capture microdissection and RNA amplification for gene expression profiling of prostate cancer. BMC Mol. Biol. 8: 25.

Li H, Chen X, Zhang F, Ma J, et al. (2007). Expression patterns of the cell junction-associated genes during rat liver regeneration. J. Genet. Genomics 34: 892-908.

Nakatani K, Kaneda K, Seki S and Nakajima Y (2004). Pit cells as liver-associated natural killer cells: morphology and function. Med. Electron. Microsc. 37: 29-36.

Norton JN (1992). Total RNA isolation by a rapid centrifugation method. Am. Biotechnol. Lab. 10: 41.

Paschos K, Canovas D and Bird N (2008). Malignant cell interactions with cells of the hepatic sinusoids mediate primarily the development of colorectal cancer liver metastasis. Ann. Gastroenterol. 21: 98-108.

Ravandi F, Talpaz M and Estrov Z (2003). Modulation of cellular signaling pathways: prospects for targeted therapy in hematological malignancies. Clin. Cancer Res. 9: 535-550.

Revel JP, Yancey SB, Meyer DJ and Nicholson B (1980). Cell junctions and intercellular communication. In Vitro 16: 1010-1017.

Scott RJ (1995). Isolation of whole cell (total) RNA. Methods Mol. Biol. 49: 197-202.

Su AI, Guidotti LG, Pezacki JP, Chisari FV, et al. (2002). Gene expression during the priming phase of liver regeneration after partial hepatectomy in mice. Proc. Natl. Acad. Sci. U. S. A. 99: 11181-11186.

Swain MG (2008). Hepatic NKT cells: friend or foe? Clin. Sci. 114: 457-466.

Taub R (2004). Liver regeneration: from myth to mechanism. Nat. Rev. Mol. Cell Biol. 5: 836-847.

Wang GP and Xu CS (2010). Reference gene selection for real-time RT-PCR in eight kinds of rat regenerating hepatic cells. Mol. Biotechnol. 46: 49-57.

Wang JZ, Du Z, Payattakool R, Yu PS, et al. (2007). A new method to measure the semantic similarity of GO terms. Bioinformatics 23: 1274-1281.

Wang WB, Fan JM, Zhang XL, Xu J, et al. (2009). Serial expression analysis of liver regeneration-related genes in rat regenerating liver. Mol. Biotechnol. 43: 221-231.

Wei H, Wei H, Wang H, Tian Z, et al. (2010). Activation of natural killer cells inhibits liver regeneration in toxin-induced liver injury model in mice via a tumor necrosis factor-alpha-dependent mechanism. Am. J. Physiol. Gastrointest. Liver Physiol. 299: G275-G282.

Xu CS, Shao HY, Liu SS, Qin B, et al. (2009). Possible regulation of genes associated with intracellular signaling cascade in rat liver regeneration. Scand. J. Gastroenterol. 44: 462-470.

Zheng ZY, Weng SY and Yu Y (2009). Signal molecule-mediated hepatic cell communication during liver regeneration. World J. Gastroenterol. 15: 5776-5783. 